\title{
Standespolitik und Einheitskasse
}

\author{
Von der FMH wird eine Stellungnahme zur Einheitskasse erwartet. Die Entschei- \\ dungsfindung darf sich keinesfalls von kurzlebigen, ärztlichen Frust-Anekdoten im \\ täglichen Umgang mit Krankenkassen leiten lassen. Sie muss sich an Grundsätzen \\ der Standespolitik orientieren.
}

\section{Walter Grete}

Von 1995-2002 Präsident der Ärztegesellschaft des Kantons Zürich AGZ

Interessenbindungen: Stiftungsrat in einer Zürcher Privatklinik

Korrespondenz:

Dr. med. Walter Grete Halden 5

CH-8184 Bachenbülach

walter[at]grete.ch

\section{Was sind die «allgemeingültigen Grundsätze der ärztlichen Standes-Politik?»}

Es sind dies die Standesregeln zur Gestaltung des Arztberufes und Leitlinien zur Optimierung des ärztlichen Berufsumfeldes. Wir nennen dies die ärztliche Gesundheitspolitik. Zur Illustration sind wichtige Inhalte im Folgenden dargestellt.

Die ärztliche Standespolitik nach innen:
- Die Aus- und Weiterbildung pflegen
- Den ärztlichen Nachwuchs fördern
- Die therapeutischen Freiheiten bewahren
- Die Qualität sichern
- Scharlatanerie und Kurpfuschertum bekämpfen
- Die Sanktionierung schwarzer Schafe ernst nehmen
- Die Zusammenarbeit im Gesundheitswesen pflegen
- Den Präventionsgedanken stärken
- Die Verantwortung des Arztes gegenüber dem Patienten
und gegenüber der mitfinanzierenden Gesellschaft fördern
Die Leitlinien ärztlicher Gesundheitspolitik:
- Die unabhängige Berufsausübung für Arzt und Ärztin
im Gesundheitswesen sichern
- Die freie Arztwahl für Patienten bewahren
- Die Kostenträger und die Leistungserbringer trennen,
Ärzte versichern nicht, Versicherungen behandeln nicht
- Nur Patienten und nicht die Versicherungen als Honorar-
schuldner akzeptieren
- Politische Tarif- und Honorargestaltung vermeiden,
betriebswirtschaftliche Tarife fordern

Der nachstehende Initiativ-Text muss in Reflexion zu den beschriebenen Grundsätzen der ärztlichen Standes- und Gesundheitspolitik gelesen werden.

\section{Ein Mega-Projekt, aber} ohne erkennbaren Projektnutzen

Im dritten Anlauf innerhalb von nur 10 Jahren will die Initiative «Für eine öffentliche Krankenkasse» die bestehenden Krankenversicherungen im Bereich der Grundversicherung enteignen und deren Aufgaben einer staatlichen Einheitskasse übertragen. Einmal mehr soll mit einer Änderung der Verfassung das Krankenversicherungsgesetz und damit das schweizerische Gesundheitswesen umgestaltet werden. Zur Zielerreichung müssten alle bestehenden Versicherungs-Verträge gekündigt, alle Versicherten infor- miert, alle bestehenden Netzwerke aufgelöst und die Systemverantwortung an einem Stichtag übergeben werden - selbstverständlich ohne Unterbruch der laufenden Zahlungen. Ein gigantisches EDV-System, das kaum aus den bestehenden EDV-Anlagen zusammengeschustert werden kann, muss erstellt und gefüttert werden und ab Stichtag funktionstüchtig sein. (Da läuten bereits die Alarmglocken.) Die geforderten kantonalen Filialstellen der Einheitskasse müssten personell ausgestattet und die Mitarbeitenden geschult sein. Die Infrastruktur muss stehen.

\section{Synergien werden zerstört}

Die Zusatzversicherungen, die Taggeldversicherungen und die Kollektivversicherungen würden ab Stichtag über getrennte Adressen laufen. Auch darüber müssten Versicherte und Leistungserbringer in der ganzen Schweiz informiert sein. Zusatzversicherte erhalten nun grundsätzlich zwei Rechnungen für Leistungen und Abrechnungen von Versicherungen, die sich aus Datenschutzgründen untereinander nicht austauschen dürfen. Kurz, die Zusammenführung des gewachsenen, eingespielten Systems zu einer öffentlichen Einheitskasse mit Filialen wäre ein überdimensioniertes Projekt mit primär sehr grossem Investitionsbedarf. Dieser wird auf über eine Milliarde Franken geschätzt. Auch jahrelange Auseinandersetzungen zur Abgrenzung der Vermögenswerte innerhalb der bestehenden Versicherungen sind voraussehbar.

Das Projekt ist riesengross, denn die Einführung einer Einheitskasse erfolgt nicht auf grüner Heide. Bei sorgfältiger Planung und entsprechenden, gewaltigen Investitionskrediten wäre das Projekt vielleicht machbar. Einem derart grossen und teuren Aufwand muss aber ein absehbarer Nutzen gegenüberstehen und dieser ist nicht erkennbar.

\section{Schweizer Bevölkerung mit Gesundheitswesen zufrieden}

Die Bevölkerung schätzt den offenen Zugang für alle Einwohner zu modernsten medizinischen Leistungen, lobt die Qualität und die freie Wahl der Leistungserbringer und lässt sich kaum je im Ausland betreuen. Aber die Schweizer Bevölkerung ist besorgt über permanent steigende Zwangsprämien. 
Die hohen Kosten - und das sind im Folgejahr die Prämien - entstehen durch die Honorare und Löhne der Beschäftigten im Gesundheitswesen einerseits und durch die Nachfrage nach Leistungen durch Patientinnen und Patienten andererseits. Dies bleibt auch bei einer Einheitskasse mit 26 Filialen unverändert, denn die Grundversicherung darf auch heute nicht gewinnorientiert arbeiten.

Ernsthafte Sparmassnahmen könnten somit nur durch die Senkung der Löhne im Gesundheitswesen und/oder durch eine Drosselung und Rationierung

\section{«Eine Einheitskasse würde Grundsätze der ärztlichen}

\section{Gesundheitspolitik tangieren.»}

Articles
interactifs
Vous souhaitez commenter
cet article? Il vous suffit
pour cela d'utiliser la fonc-
tion «Ajouter un commen-
taire» dans la version en
ligne. Vous pouvez égale-
ment consulter les
remarques de vos confrères
sous: www.bullmed.ch/
numero-actuel/
articles-interactifs/

des Angebotes erzielt werden. Die Idee, dass kantonale und regionale Einheitskassen ernsthaft günstiger arbeiten könnten als private Versicherer, ist absurd. Im Gegenteil, es würden Synergien zu anderen Versicherungszweigen entfallen. Natürlich könnten gewisse Werbekosten eliminiert und nur noch im Zusatzversicherungsgeschäft verrechnet werden. Die herbstliche Kassenwechselrunde mit lästigen Telefonaten würde abgelöst durch den geforderten Kassenwechsel beim Wohnortwechsel in eine andere Versicherungsregion. Die Kosten eines Versicherungswechsels werden im Übrigen in den Medien massiv überschätzt. Längst regelt eine kostengünstige App für die Versicherungen jeden Kassenwechsel elektronisch für wenige Franken. Die Werbekosten, die zum grössten Teil durch die $\mathrm{Zu}$ satzversicherungen gedeckt werden, liegen im Promillebereich. Von 100 Prämienfranken werden in der

\section{Wortlaut der Volksinitiative}

Die Eidgenössische Volksinitiative «Für eine öffentliche Krankenkasse» lautet: I

Die Bundesverfassung1 wird wie folgt geändert:

Art. 117 Abs. 3 (neu) und 4 (neu)

3 Die soziale Krankenversicherung wird von einer einheitlichen nationalen öffentlich-rechtlichen Einrichtung durchgeführt. Deren Organe werden namentlich aus Vertreterinnen und Vertretern des Bundes, der Kantone, der Versicherten und der Leistungserbringer gebildet.

4 Die nationale Einrichtung verfügt über kantonale oder interkantonale Agenturen. Diese legen namentlich die Prämien fest, ziehen sie ein und vergüten die Leistungen. Für jeden Kanton wird eine einheitliche Prämie festgelegt; diese wird aufgrund der Kosten der sozialen Krankenversicherung berechnet.

II

Die Übergangsbestimmungen der Bundesverfassung werden wie folgt geändert:

Art. 197 Ziff. 8 (neu) 2

8. Übergangsbestimmungen zu Art. 117 Abs. 3 und 4 (nationale öffentlich-rechtliche

Krankenkasse)

1 Nach der Annahme von Artikel 117 Absätze 3 und 4 durch Volk und Stände erlässt die Bundesversammlung die notwendigen gesetzlichen Bestimmungen, damit die Reserven, die Rückstellungen und die Vermögen aus dem Bereich der sozialen Krankenversicherung auf die Einrichtung nach Artikel 117 Absätze 3 und 4 übertragen werden.

2 Erlässt die Bundesversammlung nicht innert drei Jahren nach Annahme von Artikel 117 Absätze 3 und 4 ein entsprechendes Bundesgesetz, so können die Kantone auf ihrem Gebiet eine einheitliche öffentliche Einrichtung der sozialen Krankenversicherung schaffen.
Grundversicherung über 94 Franken für die Gesundheit der Versicherten ausgegeben. Das ist kaum zu überbieten. Auch eine Einheitskasse wird Prämien einziehen, Abrechnungen versenden und Löhne bezahlen müssen, neu dann aber ausschliesslich aus den Prämien der Grundversicherten.

\section{Die SUVA ist keine Krankenkasse}

Selbst in ärztlichen Kreisen wird die SUVA als Beispiel und Vorbild einer gutfunktionierenden staatlichen Versicherung angefügt. Dabei wird übersehen, dass die SUVA als Rentenversicherung über sehr grosse Kapitalerträge verfügen kann, dass ein grosser Teil der Administration durch die Arbeitgeber erledigt wird, und dass sich die SUVA im gewünschten Wettbewerb der Unfallversicherer bewähren muss. Exakt dieser Wettbewerb würde der neuen Monopolversicherung aber fehlen.

\section{Die Einheitskasse: übermächtiger Player in der Gesundheitspolitik}

Neben all den kostspieligen technischen Umsetzungsproblemen würde eine Einheitskasse das Patienten-Arztverhältnis tangieren, die unabhängige Berufsausübung einengen und die Ärzteschaft in die Versicherungsverantwortung zwingen. Eine staatliche Einheitskasse würde das Gesundheitswesen zusätzlich politisieren. Der Druck durch den Monopolisten auf missliebige Leistungserbringer wäre enorm. Der im Abstimmungstext geforderte Einsitz der Ärzteschaft in die Führungsgremien der schweizerischen Einheitskasse würde das Prinzip der Trennung zwischen Kostenträgern und Leistungserbringern verletzen. Wir Ärzte müssten für alle Sparmassnahmen im Gesundheitswesen institutionalisiert geradestehen und allenfalls gar gegen die Bedürfnisse der Patienten und gegen eigene Honoraranliegen auftreten.

Ein Gesundheitswesen ohne Versicherungen ist in der Schweiz undenkbar und wäre auch für die Ärzteschaft katastrophal. Die Vielfalt der Versicherungen ermöglicht eine angepasste, freie Zusammenarbeit zwischen Patienten, Versicherungen und Ärzten, zum Beispiel im Wettbewerb innerhalb von alternativen Versicherungs-Modellen. Im Monopol einer Einheitskasse wird Zusammenarbeit jedoch zum Diktat, denn jede Alternative wird fehlen. Wer Versicherungen verstaatlichen will, wird auch vor der Verstaatlichung der ärztlichen Infrastrukturen im Gesundheitswesen kaum haltmachen. Cavete!

Ein derart aufwendiger Systemwechsel müsste Verbesserungen in das schweizerische Gesundheitswesen bringen, die ins Auge springen. Die Analyse dieser Initiative lässt aber keine Verbesserungen erkennen.

Ärztinnen und Ärzte sind wichtige Meinungsbildner auch bei Fragen rund um das Gesundheitswesen. Nehmen wir zeitgerecht gegen diesen sinnlosen Schritt zur Teilverstaatlichung des schweizerischen Gesundheitswesens Stellung! 